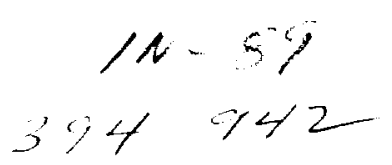

\title{
Developmental cryogenic active telescope testbed, a wavefront sensing and control testbed for the next generation space telescope
}

\author{
Claudia M. LeBoeuf", Pamela S. Davila ${ }^{a}$, David C. Redding ${ }^{\mathbf{b}}$, Armando Morell ${ }^{\mathbf{a}}$, Andrew E. Lowman \\ Mark E. Wilson', Eric W. Young ${ }^{a}$, Linda K. Pacini, Dan R. Coulter \\ a - NASA Goddard Space Flight Center (GSFC), Greenbelt, MD 20771 \\ b - Jet Propulsion Laboratory (JPL), 4800 Oak Grove Drive, Pasadena, CA 91109
}

\begin{abstract}
As part of the technology validation strategy of the next generation space telescope (NGST), a system testbed is being developed at GSFC, in partnership with JPL and Marshall Space Flight Center (MSFC), which will include all of the component functions envisioned in an NGST active optical system. The system will include an actively controlled, segmented primary mirror, actively controlled secondary, deformable, and fast steering mirrors, wavefront sensing optics, wavefront control algorithms, a telescope simulator module, and an interferometric wavefront sensor for use in comparing final obtained wavefronts from different tests. The developmental cryogenic active telescope testbed (DCATT) will be implemented in three phases. Phase 1 will focus on operating the testbed at ambient temperature. During Phase 2, a cryocapable segmented telescope will be developed and cooled to cryogenic temperature to investigate the impact on the ability to correct the wavefront and stabilize the image. In Phase 3, it is planned to incorporate industry developed flight-like components, such as figure controlled mirror segments, cryogenic, low hold power actuators, or different wavefront sensing and control hardware or software. A very important element of the program is the development and subsequent validation of the integrated multidisciplinary models. The Phase 1 testbed objectives, plans, configuration, and design will be discussed.
\end{abstract}

Keywords: NGST, active optics, wavefront sensing, wavefront control, segmented optics, hexapod, stewart platforms

\section{INTRODUCTION AND BACKGROUND}

The next generation space telescope (NGST) plans to use optical controls to clean up errors from a variety of sources, such as deployment errors, fabrication errors, thermal deformations, and vibrations from spacecraft hardware. Building in a high degree of on-orbit adjustability dramatically reduces requirements in these traditionally difficult and expensive areas'. In all three of the design studies for NGST, the ability to sense and control the wavefront from the optical system to achieve diffraction limited imaging on-orbit was a key feature. The challenges presented by a space-based observatory are different than for many large aperture ground-based observatories. To some degree, the design is easier because elaborate gravity compensation schemes do not have to be implemented (though ground testing becomes a major concem) and compensation for degraded imaging due to atmospheric turbulence is not a concern. On the other hand, the cold environment of space mandates the development of components and systems that will operate at low temperatures, typically 30 degrees Kelvin (for the L2 LaGrange point orbit). Launch vehicle size limits either constrain the maximum diameter or dictate a segmented primary mirror. The wavefront sensing and control system must be able to detect and control optical wavefront errors resulting from thermal gradients and vibrational modes coupled to the system structural design and the spacecraft design.

NASA will develop a series of ground testbeds and validation flights to demonstrate the technologies of deployable and adjustable lightweight optics, cryogenic optics and mechanisms, passive cooling, and on-orbit, closed loop wavefront control techniques ${ }^{2}$. The developmental cryogenic active telescope testbed (DCATT) is being developed primarily to address the systems and performance issues of applying closed loop wavefront control techniques to a system which includes all of the functional elements which are presently envisioned for a successful NGST mission. These include a segmented actively controlled primary, an actively controlled secondary, a deformable mirror, a fast steering mirror, and wavefront reconstruction and control algorithms.

The DCATT project will be implemented in three phases. The first phase will focus on getting an ambient system operating. The telescope primary mirror will be segmented and actuated in six degrees of freedom but the segments will be rigid, not figure controllable. It is planned that the Phase I testbed will be integrated and operational early in 1999. During the second phase a cryocapable segmented telescope will be developed and cooled to cryogenic (liquid nitrogen) temperature. This will also require cryocapable segment actuation systems. Unless the project is provided with figure controllable cryocapable optic 
segments for test from outside sources at that point in time. the primary mirror segments will be rigid. It is planned that the system would be integrated and operntional early in $200 \mathrm{~L}$. During Phase III, it is planned that technology elements developed by industry through the NGST technology development program will be integrated into the system. This might include such things as figure controllable. cryocapable primary mirror segments, cryocapable flight-like actuators and/or sensors, and different wavefront sensing and control techniques, devices, and algorithms. The timing of this phase depends on the availability of the technology and the hardware. Portions of it may be phased in during Phase I or II of the program. depending on the applicability, ease of integration, and priority of other tests. At this time, it is planned that the integration of such technology will begin in 2002.

\section{OBJECTIVES AND REQUIREMENTS}

The primary objective of the DCATT project is to represent in hardware an optical system that incorporates all of the functional elements envisioned in an NGST active optical system and to investigate the viability and efficiency of candidate wavefront sensing and wavefront control techniques and algorithuns. Essentially, it is intended to explore the ability of a candidate technique to reduce large wavefront errors to diffraction limited values and to document the envelope of controllability, or in other words, robustness, of the system. The system is being designed to be diffraction limited at 2 microns to simulate the performance requirements of the NGST system.

A second objective of equal importance is the development and validation of integrated computer models of the DCATT system. It is through the incorporation of these modeled effects into the NGST models and simulations that system results from DCATT can be scaled for use in predicting NGST performance ${ }^{3}$. It must be emphasized that the DCATT hardware design has not been intended to represent a scaled down version of future NGST hardware. Details of the various NGST design studies have been examined in order to design to scales that adequately represent the functions of elements proposed for the NGST optical system without introducing all of the testing difficulties of full scale elements. Of key importance will be the characterization of each component of the testbed prior to integration.

Some additional goals which fall out of the objectives include investigating the transitions and interactions between the functional modes of coarse segment alignment and fine segment alignment and phasing; investigating the interactions between, phasing of, and requirements of a deformable mirror and a fast steering mirror as elements of the optical path; investigating issues of fabrication, testing, and operation of a segmented active telescope, at ambient and at cryogenic temperatures; and identifying key NGST optical system level issues.

For the GSFC concept presented in the summer of 1996, the wavefront sensing and control scheme included phase retrieval as a baseline method. Image enhancement and phase retrieval methods have been proven on $\mathrm{HST}^{4}$. One of the first goals of the testbed effort will be to prove out this method for a segmented aperture optical system. Another goal of the testbed activity is to have a calibrated optical system that can then provide a standard for future testing of other wavefront sensing and control techniques.

The top level requirement for the DCATT optical and control system is to demonstrate that diffraction limited imaging performance can be achieved at a wavelength of 2 microns under closed loop control. This translates to a corrected wavefront error goal of $1 / 14$ wave root mean square (rms) at 2 microns or $1 / 4.45$ wave $\mathrm{ms}$ at 633 nanometers (more detail on the optical design ${ }^{5}$ and the error budget ${ }^{6}$ can be found in separate papers). There must be a low vibration environment in order to do interferometric measurements as well as for image stability. A reflective optical design and a minimum number of elements were desired such that infrared testing could be done at a later date. The aft optics are as much as possible divided into modular units (e.g. the source module or the telescope simulator module) which will be built up and characterized on their own platforms and then be kinematically mounted to the optic bench. As well as allowing the various characterization tasks to occur in parallel between several organizations, this allows the incorporation of different optical configurations for alignment, calibration, and open and closed loop testing of the system. Commercial components were selected wherever possible for cost and schedule reasons.

\section{TESTBED CONFIGURATION}

Figure 1 shows the overall testbed configuration for the ambient phase. A steel base supported on six vibration isolation mounts provides the foundation for the telescope assembly and for the aft optics bench. The base will be located on an isolated granite slab in a cleanroom environment. 
The main components of the telescope assembly are a segmented parabolic primary mirror (PM), a hyperbolic secondary mirror (SM), 6 degree of freedom (DOF) actuation systems (hexapods) for each PM segment and the SM, a metering tower between the PM and the SM, and a 3-DOF optical autocollimating flat. The aft optics bench contains the Source module (Source), a deformable mirror (DM), a fast steering mirror (FSM), a dispersed fringe sensor (DFS) for coarse segment phasing, a translating CCD camera for fine segment and DM alignment and phasing, a telescope simulator module (TSM) that will generate specific calibrated wavefront aberrations, and an interferometric wavefront sensor (IWFS) for the purpose of ineasuring and comparing the final achieved wavefront. The DFS and CCD camera together make up what is called the wavefront sensor (WFS). The testbed will be surrounded by a shroud to block air currents and provide some acoustic damping. Salient features of the components will next be described.

\subsection{Telescope Design}

After reviewing a number of different telescope designs, a two mirror Cassegrain design was settled on for simplicity and ease of fabrication, alignment and test ${ }^{5}$. There were several reasons behind the vertical orientation. One benefit is the greater horizontal compactness, which allows the whole system to be easily tied together on a vibration isolation frame. Other reasons are the desire for an even gravitational force over the mirror surfaces, minimization of side loads on the actuators and on the metering structure without resorting to a complex off-loading design, and the desire to eventually have a vertical configuration for the cryogenic phase such that vertical temperature stratifications in the chamber would have a minimal effect across the face of the mirror. Because of the vertical orientation, it was necessary from a practical standpoint to include as well an autocollimating flat and to double pass the light through the telescope portion.

The parabolic primary mirror design consists of a ring of 6 light weight hexagonal segments arranged around a central hexagon segment. The size and shape of the segments was chosen for ease of fabrication and minimization of gravity sag, while being large enough to be of reasonable scale to the actuators and provide good angular step resolution through spacing of the actuators. If a Shack-Hartmann WFS technique were ever tested, the straight edges could accommodate edge sensors. The edges of the segments are separated by $6-\mathrm{mm}$ gaps. The secondary mirror is hyperbolic. Table 1 below shows the first order design parameters and specifications of the telescope. The clear aperture of the $\mathrm{f} / 2$ primary mirror is approximately 90 $\mathrm{cm}$. The design goal for the telescope is to achieve diffraction limited imaging over a 2-by-2 arcminute field of view. Both mirrors, as well as their baseplates and interconnecting structures, are all being fabricated using T6061 T65I aluminum (age hardened, mechanically stress relieved). As presently designed, the metering structure between the primary and secondary mirror has a first resonant frequency (a bending mode) of approximately $50 \mathrm{Hertz}$.

Figure 2 shows the segmented primary mirror assembled on the baseplate in the operating condition with the 6-DOF actuation systems (termed "hexapods") on each segment. Each hexapod has 6 Burleigh PZT inchworm devices each having over 6 millimeters of linear travel in 3 to 4 nanometer steps. Also incorporated into the Burleigh actuators are absolute encoders with a resolution of 0.5 micrometer. The 6-DOF's are independent in that the system can provide displacement of the segment in one DOF without displacement in any other DOF (there will be some minute coupling based on step size tolerances). In order to obtain this displacement, all 6 actuator positions must generally change. Table 2 lists the segment theoretical resolutions and possible ranges for movement in tip, tilt, and piston. Table 3 shows two possible maximum range positions of 6-DOF motion of a segment. As seen in Figure 3, each leg of the hexapod has three flexures, a bending flexure between the actuator and the segment and one rotational and one bending flexure between the actuator and the base. A flexure design was chosen over a friction joint design in order to eliminate unpredictable friction effects on motion and to ease later adaptation to a cryogenic environment. The flexure stiffnesses were chosen to optimize between minimizing the stresses at the mirror surface due to actuator forces and maximizing the resonant frequency of the structure.

The Zerodur autocollimating flat will be mounted on a nine-point Hindle mount, which will in tum be mounted to a motorized jackscrew system having tip, tilt, and piston control. This feature allows the flat to be directed off-axis in order to align and phase mirror segments and possibly to experiment with off-axis sources and phase diversity methods in the future. The flat will be polished to a $1 / 10$ wave rms wavefront and coated with aluminum with $\mathrm{MgF}_{2}$ overcoat $^{5}$. The maximum gravity sag has been analyzed to be less than 40 nanometers peak-to-valley near the mounting points, with the effect dropping off rapidly away from these points.

\subsection{Primary Mirror Fabrication}

Some details of the fabrication of the segmented, diamond tumed, nickel-plated aluminum primary mirror may be of interest to the reader. The PM was fabricated as seven hexagon shaped seginents. 30 millimeters flat to flat, cut individually from the 
same boulc of aluminum T6061 T65I, and fully machined except for the front surface, which was lef as a flat prior to diamond turning. The back pocket depth varies, leaving a constant mirror shell thickness of 3 millimeters supported by an isogrid pattern of pockets of approximately 1 inch inscribed diameter (Figure 4 ). The thin-walled tube between the segment back surface and the mounting flange is designed to isolate the segment front surface from loads at the mounting flange and is sized in diameter to minimize gravity sag and provide bending stiffness against centrifugal forces during diamond turning. The final weight of each segment will be approximately 5 pounds. Dimensional inspection prior to and after the removal of material from the front surface of a test piece revealed an approximately .005 inch "saddle" deflection pattern. It was desired to totally relieve the stresses in the segments and recut the mating surfaces flat prior to dianond turning of the front surface. As it was determined that the stresses the segments would experience during fabrication (rotation at up to 300 revolutions per minute) and operation were much lower than the annealed strength of aluminum (10,000 pounds per square inch, psi), each segment was heat treated to the fully annealed condition in order to relieve stresses in the material to the highest degree possible. It is interesting to note that another material choice given the annealing treatment would have been aluminum 5083 , which has lesser properties than T6061 in the hardened condition, but has a strength of 16,000 psi in the annealed condition. The mounting flange of each segment was diamond turned flat to within 8 microns as were the mounting surfaces of the aluminum T6061 T651 baseplate.

The segments will be mounted with a gap of 6 millimeters between each edge and pinned for location. Also in the mirror fabrication assembly are "surround" pieces that filled in the remainder of a circular mirror as well as an extra annulus around the outer edge. This is done for two reasons. The diamond turning operation requires a relatively continuous cutting surface, though the 6-millimeter gaps are not expected to present a problem. Secondly, the rigid surround provides the polishing tool a non-deflecting surface that will prevent it from applying excessive force to the edges of the segments. After assembly of all parts on the baseplate, the parabola will be cut into the entire surface and the surface diamond turned at Oak Ridge National Laboratory. The segments and surround pieces will be removed and electrolessly plated with approximately 127 microns of nickel. That thickness was chosen to allow refiguring in the nickel after allowances for distortion of the segments due to the mismatch in coefficient of thermal expansion between nickel and aluminum and tolerances of the pinned reassembly process, which could add a height variation on segment to segment surfaces. Analysis showed the effect of the nickel cooling from the bath temperature of $\mathbf{8 5}$ degrees Celsius to room temperature to be a flattening of the segment with the edges remaining in a fairly constant position and the center of each segment surface deflecting upward approximately 33 microns. The possibility of plating the entire assembly as a unit was investigated and rejected. Besides having to find a facility with large enough tanks to plate, clean, and etch a large object, there are risks involved with plating a high volume/surface area object, mainly the rapid changes of bath chemistry it causes which need to be counteracted. The pinning tolerances will keep the reassembled height variation to less than 15 microns. The nickel-plated assembly will be diamond turned and then polished to a final surface figure of less than 0.1 wave rms at 633 nanometer and a surface roughness less than 80 Angstroms rms. The fabrication baseplate will also be used during operation and will provide an original reference for the assembled mirror.

\subsection{Aft Optics Bench Design ${ }^{5}$}

Figure 5 shows a top view of the layout of the aft optics bench. The Source module is located near the telescope focus and consists of a white light source and a 633 nanometer laser for use with the DFS and later with the IWFS. There is also space reserved for potentially adding two more laser frequencies (612 and 543 nanometers) to assist in doing multiple wavelength interferometry. The purpose of the TSM is to have a source of calibrated wavefront errors that supplements those provided by the segmented telescope system. It includes a small ( 69 active actuators) deformable mirror for generating continuous aberrated wavefronts and a set of discrete transmissive phase plates (windows with discrete steps polished in them to generate piston in the wavefront) mounted on a filter wheel. Its first use will be in calibrating and testing the accuracy of the DFS and the WFS camera.

The DM requirement is at least 1 actuator per $50 \mathrm{~mm}$ spacing across the PM, which leads to a requirement of at least 18 actuators across the DM on 7 to $8 \mathrm{~mm}$ centers. The Xinetics 349 actuator device selected utilizes PMN actuators having a 4 -micron range of travel. The FSM requirements are still under investigation, but it is thought that the device bandwidth will be on the order of a few hundred Hertz, with a range adequate to handle drift and DC offset errors as well as any low frequency modes that may be excited in the metering structure. A quad cell will provide closed loop feedback to the FSM.

Three methods of wavefront sensing are represented in the layout. Coarse segment phasing will be accomplished using the DFS, which can be rotated in and out of the optical path. The primary fine phasing and alignment wavefront sensing will utilize the CCD camera, mounted on a translation stage for focus and defocus images. The third method represented is the IWFS. It can be located in two different locations on the testbed to perform different functions. The IWFS will quantify the 
wavefront error, including piston, at various stages of the wavefront sensing and control process. These measurements will be used to evaluate the degree of wavefront correction, as well as the accuracy of different phase retrieval algorithms or other wavefront sensing approaches. The baseline design for the IWFS is a modified phase-shifting Mach-Zehnder interferometer, with a pinhole in one ann used to generate the reference beam'. Multiple wavelength interferometry will be utilized to increase the effective measurement wavelength, providing a means of correctly reconstructing moderate wavefront piston from modulo $2 \pi$ phase data ${ }^{8}$. The IWFS may eventually be tried as the controlling wavefront sensor for the system.

\section{3.t Control System}

Figure 6 shows a functional block diagram of the system. The various device platforms shown will be connected via a dedicated local network connection and the Sun will also have an external network connection. The Sun station will be the primary analysis center, feeding position commands to the computers controlling the devices (two PC's and one Mac). The software environment on the Sun will center around Matlab and the integrated modeling tools being developed for NGST (MACOS and IMOS, described later), while the device controllers primarily utilize Labview software and drivers.

The outline of the planned baseline control sequence for segmented telescope testing is as follows:

1. Capture secondary and center segment using the imaging camera and segment and SM hexapod actuators. This procedure uses the white light source and a conical scan of the secondary to find the light.

2. SM alignment using the imaging camera and the SM hexapod actuator. At this point, the SM would be scanned in focus and coma to obtain the best position.

3. Single segment capture using a procedure similar to above looking at an off-axis spot. Do for each outer segment using imaging camera, and segment hexapod actuators.

4. Single segment alignment in six DOF for each outer segment in turn.

5. Pupil alignment phase. Use pupil imaging mode ${ }^{5}$.

6. Segment cophasing using the dispersed fringe sensor, imaging camera, and segment hexapods. The goal of this step is to phase the segments to within one wave. Basically, the imaging camera and white light source are used to coalign the outer segment to the center segment by overlaying the spots at the primary field of view. After this, the DFS is rotated in and used to observe fringes in dispersed, overlying spots as the two segments are moved in piston to find the best alignment position as computed by fringes.

7. Segment cophasing through white light interferometry, using the imaging camera and segment hexapods. This could be used to sharpen the image if necessary, verifies the DFS cophasing, and makes a higher accuracy measurement of absolute piston error.

8. Phase retrieval wavefront sensing using the imaging camera, filter wheel, segment hexapods, and deformable mirror actuators. Images will be taken with the camera at a matrix of several focus positions. The baseline analysis method will be by a modified Gerchburg-Saxton method to compute the best estimation of wavefront, after which appropriate segment and DM actuator commands would be calculated and implemented. This step would take the system from less than one wave of error to diffraction limited performance ${ }^{6}$.

More details on the wavefront reconstruction and control analysis techniques are presented in a separate paper ${ }^{1}$.

\section{EXPERIMENT PLAN}

The first line of study will be to explore the robustness, or envelope of controllability, of the system using the wavefront sensing techniques and wavefront control algorithms outlined earlier in this paper. The first step will be to start with the manually aligned system and obtain a diffraction-limited system using all 6 DOF on the segments and using the DM. After the baseline WFC system obtains its optimum solution. the IWFS will be used to score the solution. 
Assuming that the control system has done an acceptable job under these conditions, the following investigations are planned, not necessarily in the order stated.

- After using the 6 DOF systems to misalign the segments in various ways, investigate the ability and efficiency of the control system to correct the wavefront using 3,4,5, or 6 DOF of correction. Score each final solution using the IWFS. Document the relative performance of the coarse phasing versus the fine phasing, in other words "How far will the coarse phasing mode take us?"

- Investigate the stability of the system to hold the wavefront over time in the characterized laboratory environment.

- Investigate the performance difference of small changes to the control algorithm.

- Experiment with off-axis light sources (via tilting of the autocollimating flat) and phase diversity methodologies.

- Investigate the use of the IWFS in the mode of the controlling wavefront sensor.

\section{MODELING PLAN}

A very crucial element to the testbed project is a detailed integrated model predicting the behavior of the optical control system. It is through the validation and use of this model that results from the DCATT testbed can be scaled for use eventually on an NGST sized system. The integrated modeling tools include IMOS (Integrated Modeling of Optical Systems), and MACOS (Modeling and Analysis for Controlled Optical Systems), two powerful tools for investigating the performance of an active optical system ${ }^{3}$. It is planned to model and characterize every component in the testbed. Special attention will be given to developing accurate models of the Wavefront Sensing devices, including the CCD detector and the DFS, the DM, PM, SM and actuation devices, including actuator influence functions, the thermal and mechanical environment, and the FSM. All of these modeled effects will be input to a simulation that mimics the behavior of the real system, including simulations of the coarse alignment using the DFS and all steps of the fine alignment and phasing. The simulation takes the current position of the optical surfaces and iteratively calculates an optimum estimate of the wavefront, from which new actuator position commands are derived and sent. This process would continue until it was determined that no further increase in performance could be obtained. The final goal will be to have the simulation steps and results match the steps and results of the real experiment.

\section{DCATT'S ROLE IN THE DEVELOPMENT OF NEXUS AND NGST}

The DCATT program will have a number of products that will support the NEXUS and NGST programs. NEXUS is a flight testbed program for NGST which will test wavefront sensing and control methods on a sub-NGST-scale segmented primary telescope in a space environment?

In the area of wavefront sensing and control (WFS\&C), DCATT will of course furnish general information on the applicability and performance of different wavefront sensing techniques and wavefront control algorithms. It may however be able to provide more concrete WFS\&C products. These might be in the form of tested algorithms and software architecture and code that could be rolled into the ensuing flight missions with relative ease. At the very least, prototype NEXUS and NGST software could be tested using the DCATT hardware.

Another concrete product of the testbed will be a set of validated integrated models. These could be scaled and augmented to the size and environment of the flight mission and used to simulate various control scenarios.

Another service of the testbed that will benefit the NEXUS and NGST missions is as a testbed for prototype hardware such as cryogenic actuators or figurable cryogenic mirror segments. Implementation in the testbed will not only provide information on the performance of the device in the system, but also on its effects on the overall wavefront control scheme.

\section{DCATT'S ROLE AS A FACILITY FOR THE SCIENTIFIC AND INDUSTRIAL COMMUNITY}

While DCATT's primary role is as a testbed for a narrow field of NGST directed technology options, the possibility exists for an expanded role that could benefit a broader field of scientific and industrial partners. This would most likely take the 
form of replacing elements in the testbed system with elements developed by the user. These could be such things as cryogenic actuation systems and/or figurable mirror segments, deformable mirrors, wavefront sensing devices and/or techniques, wavefront control algorithms and code, fast stcering mirror technology, or cryogenic high resolution, high range position sensors.

Once the investigation of the baseline methods are accomplished. it is proposed to do similar studies of other promising WFS candidates, such as interferometric (point diffraction, shearing), or Shack-Hartmann for example, and other WFC processes. The DCATT project would hope to have the participation of the industrial and scientific community in this broadened area of study.

\section{CONCLUSION}

A testbed has been described that will represent in hardware an optical system that incorporates all of the functional elements envisioned in an NGST active optical system. The testbed's primary function is to investigate the viability and efficiency of candidate wavefront sensing and wavefront control techniques and algorithms on a segmented active optical system, both under ambient and cryogenic environments. The initial plase of testing will be ambient and will focus on the technique of phase retrieval. Other methods will be tested and compared as time allows. It is hoped to eventually incorporate industry and science community developed components that will enable enhanced performance to be proven out.

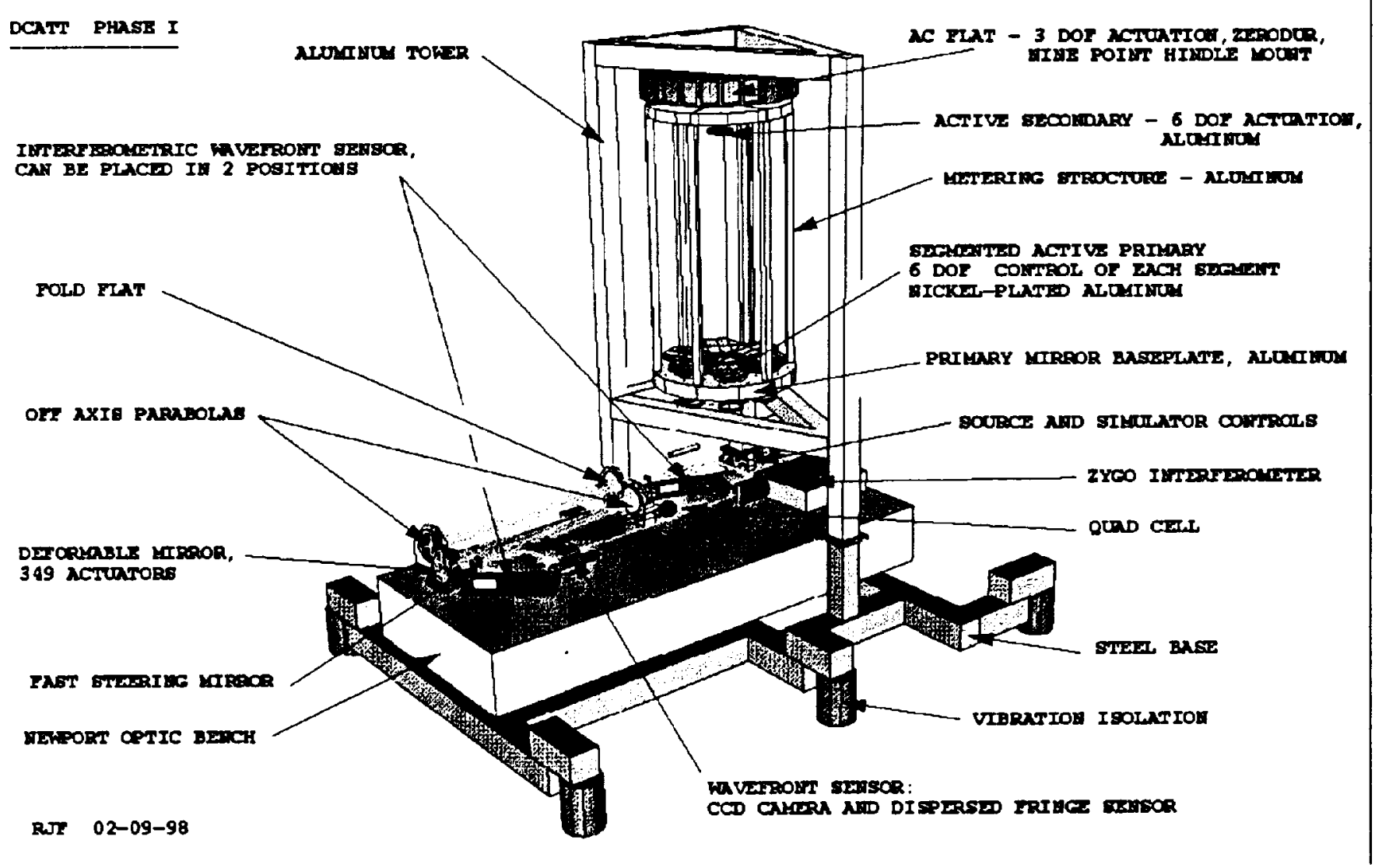

Figure 1 - DCATT Overall Configuration 


\begin{tabular}{|l|l|}
\hline Telescope & Parameter \\
\hline Primary mirror clear aperture diameter & $903 \mathrm{~mm}$ (Aperture stop at primary mirror) \\
\hline Primary mirror f/number & 2.2 \\
\hline Telescope f/number & 16.6 \\
\hline Telescope back focal distance (from primary vertex to image) & $1250 \mathrm{~mm}$ \\
\hline Primary to secondary intervertex distance & $1617.637 \mathrm{~mm}$ \\
\hline Secondary mirror clear aperture diameter & $174 \mathrm{~mm}$ \\
\hline Secondary mirror magnification & 7.5 \\
\hline Overall Length (from secondary to paraxial image plane, unfolded) & $2867.639 \mathrm{~mm}$ \\
\hline Design wavelength & $632.8 \mathrm{~nm}$ \\
\hline Exit pupil diameter & $193.5 \mathrm{~mm}$ \\
\hline Exit pupil location(distance from paraxial image plane to secondary) & 3214 (mm) \\
\hline Total field of view & $3.16 \times 2.10$ arcminutes \\
\hline Primary mirror hole cutout diameter (for unvignetted field of view) & $84 \mathrm{~mm}$ \\
\hline Obscuration ratio & 0.2 \\
\hline Aft Optics & \\
\hline Overall length (from telescope image to OAP 1 clear aperture center) & $2039.139 \mathrm{~mm}$ \\
\hline Overall Width & $944 \mathrm{~mm}$ \\
\hline OAP Focal Length & $2032 \mathrm{~mm}$ \\
\hline OAPl and OAP2 clear aperture diameters & $164 \mathrm{~mm}$ \\
\hline Fold flat diameter & $155 \mathrm{~mm}$ (Circular, Active Area) \\
\hline DM Diameter & $137 \mathrm{~mm}$ (Circular, Active Area) \\
\hline FSM Diameter & $56 \mathrm{~mm}$ (Circular, Active Area) \\
\hline System & \\
\hline F/number & 16.6 \\
\hline Exit Pupil Diameter & $438 \mathrm{~mm}$ \\
\hline Exit Pupil Location (distance from CCD to final exit pupil) & $7300 \mathrm{~mm}$ \\
\hline
\end{tabular}

Table 1. First order design parameters for the D CATT telescope, aft optics, and system

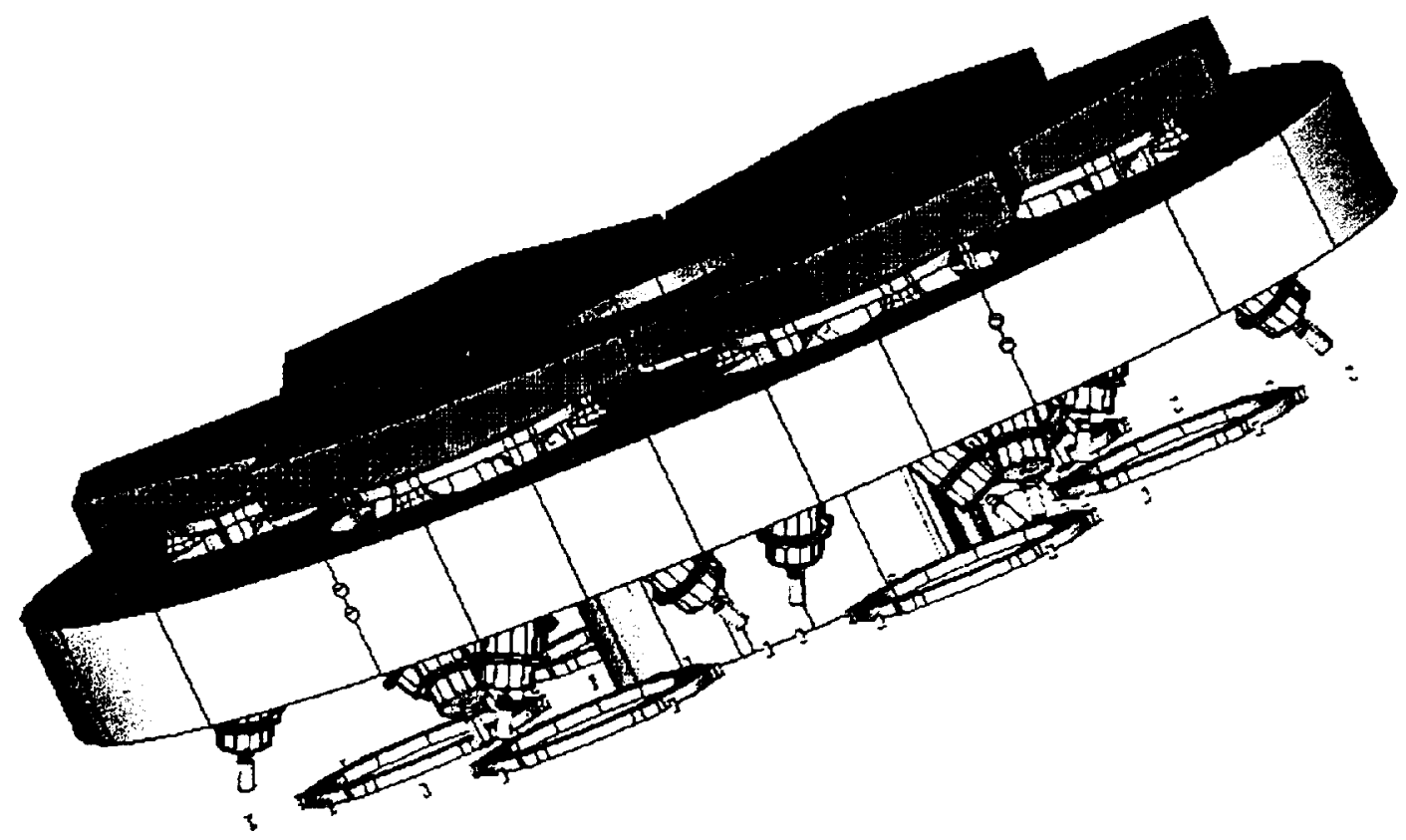

Figure 2 - Segmented primary mirror assembly with hexapod actuation systems 


\begin{tabular}{|l|l|l|}
\hline Criterion & Requirement & Current Design \\
\hline & & \\
\hline Tip/tilt resolution & \pm 0.01 arc-sec & $\pm \sim 0.002$ arc-sec single step resolution \\
\hline & & \pm 0.25 arc-sec absolute encoder knowledge \\
\hline & & \\
\hline Tip/tilt range & \pm 0.5 degree & \pm 1.0 degree with \pm 1.0 millimeter piston \\
\hline & & \pm 1.7 degree with \pm 0.5 millimeter piston \\
\hline & & \pm 2.0 degree maximum range \\
\hline Piston resolution & & \\
\hline & \pm 0.1 micrometer & $\pm \sim 0.005$ micrometer single step resolution \\
\hline & & \pm 0.6 micrometer absolute encoder knowledge \\
\hline Piston range & & \\
\hline & \pm 0.05 millimeter & \pm 3.4 millimeter maximum range \\
\hline
\end{tabular}

Table 2 - Resolutions and ranges of primary mirror segments in 3 DOF

\begin{tabular}{|l|l|l|l|}
\hline Case 1 & Tip: +1.44 degree & Tilt: -1.44 degree & Piston: -0.5 millimeter \\
\hline & Spin: -0.4 degree & X-decenter: -0.5 millimeter & Y-decenter: +0.5 millimeter \\
\hline & & & \\
\hline Case 2 & Tip: +0.56 degree & Tilt: +0.56 degree & Piston: +0.5 millimeter \\
\hline & Spin: -1.0 degree & X-decenter: +1.0 millimeter & Y-decenter +1.0 millimeter \\
\hline
\end{tabular}

Table 3 - Sample positions at edge of 6-DOF motion envelope

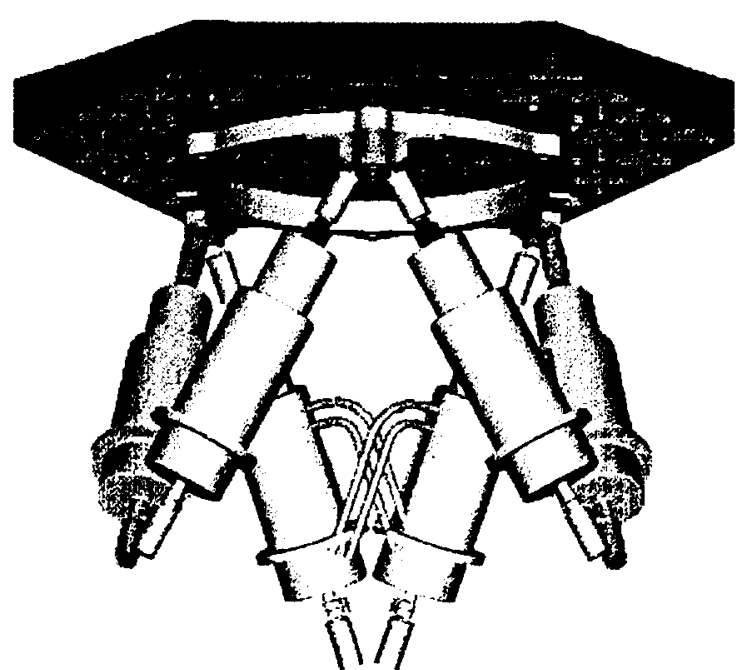

Figure 3 - PM segment on hexapod actuation system

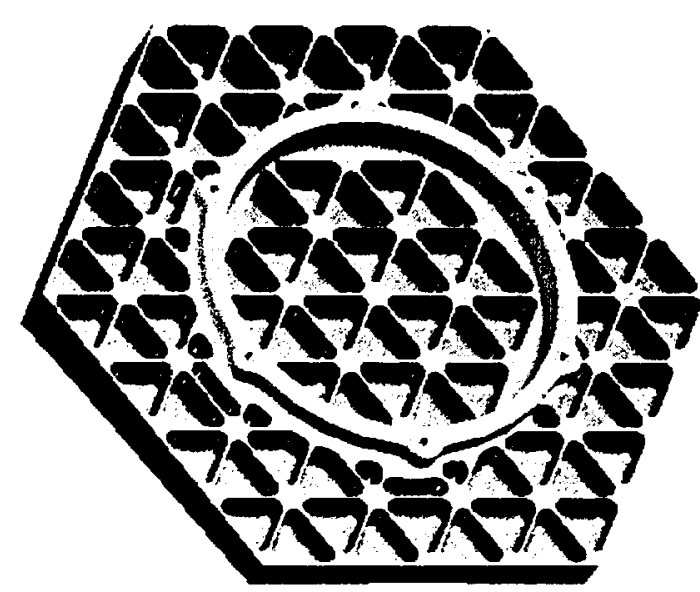

Figure 4 - PM segment back side 


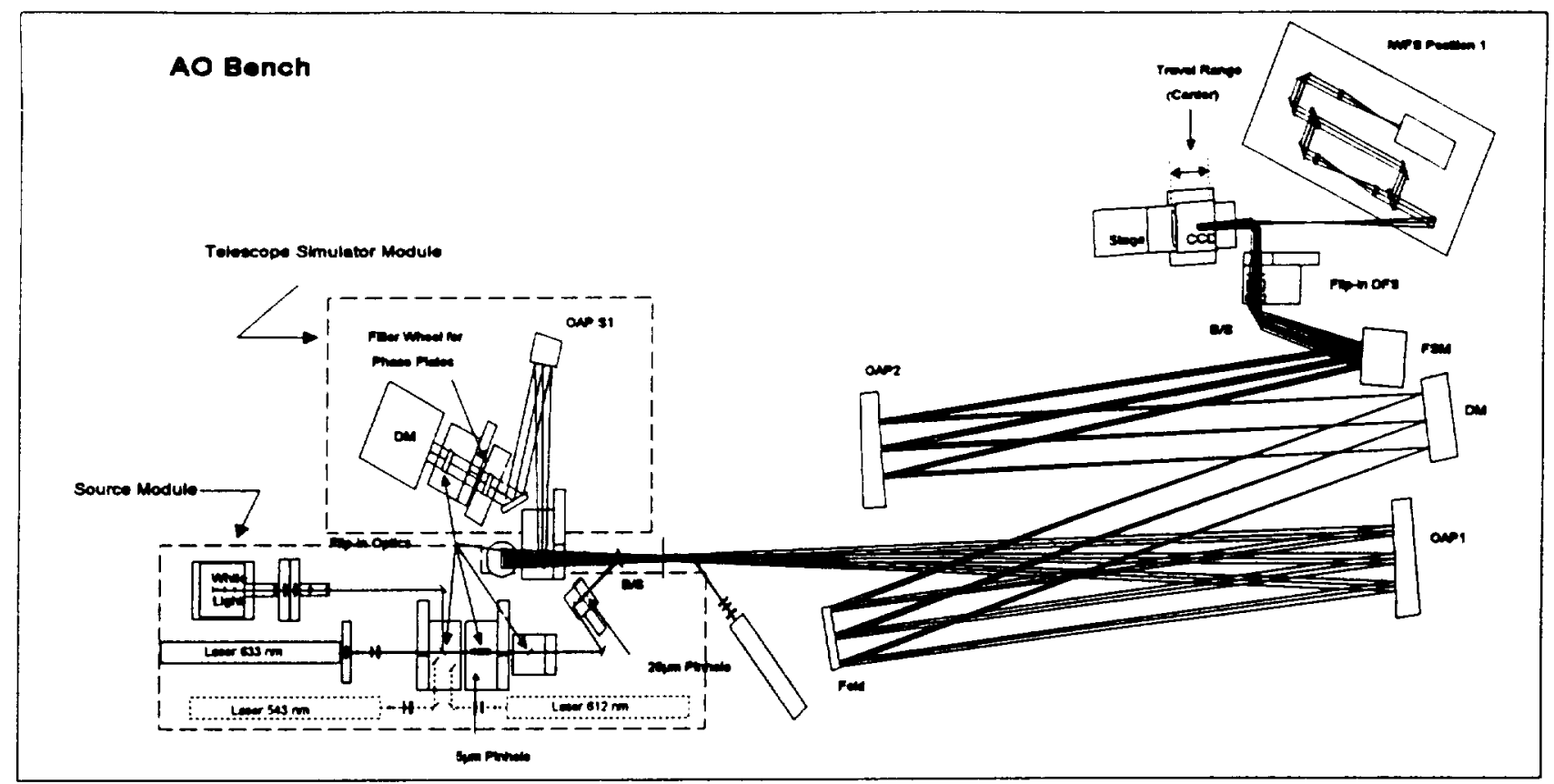

Figure 5 - Optical layout

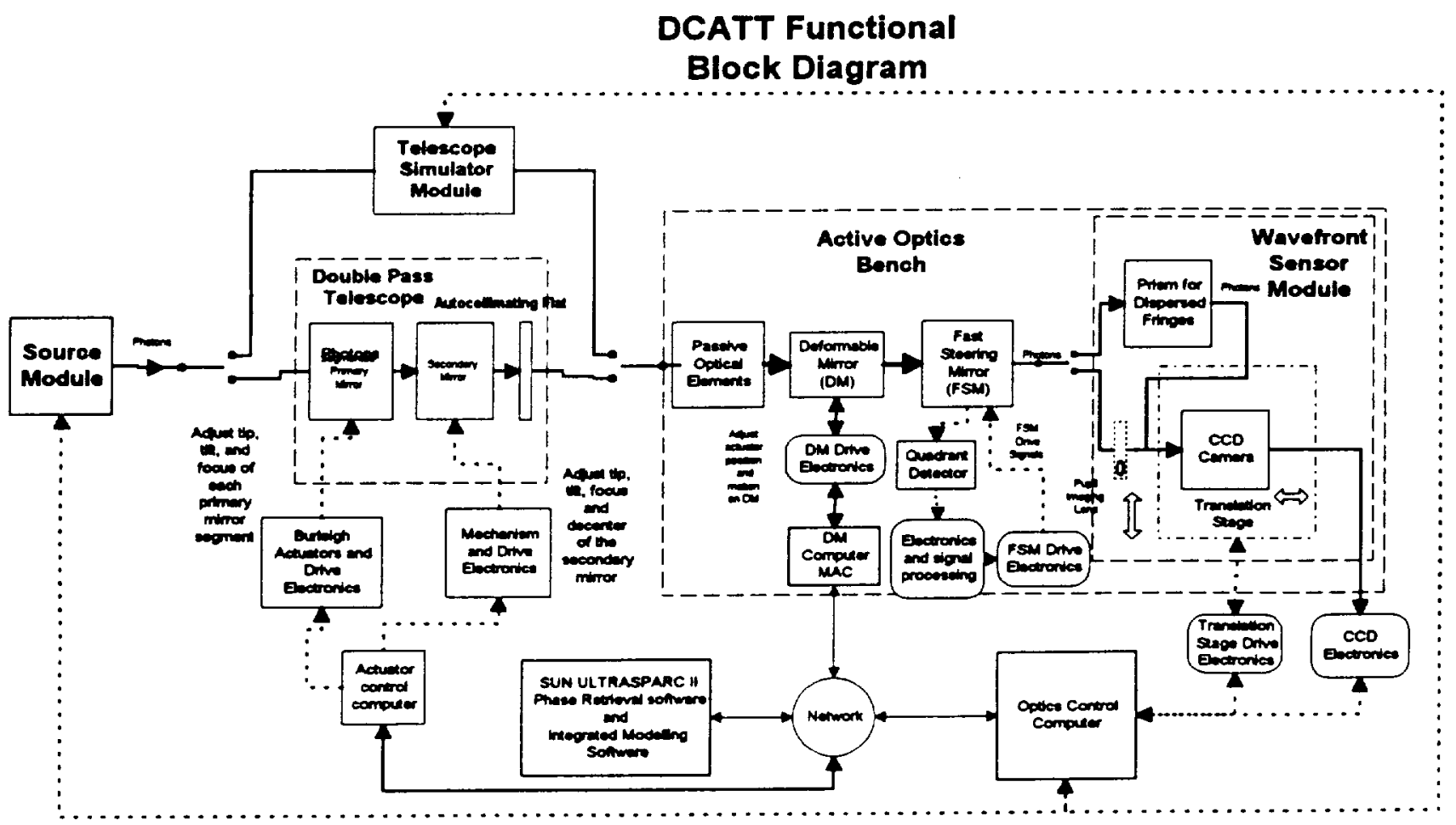

Note: Although not shown, the source module and the telescope simulator module

are physically located on the active optics bench

Figure 6 - Functional block diagram 


\section{REFERENCES}

1. D. C. Redding, S. A. Basinger. A. E. Lowman, A. Kissil, P. Y. Bely, R. Burg, G. E. Mosier, M. Femiano, M. E. Wilson, D. N. Jacobson. J.Rakoczy, "Wavefront sensing and control for a next generation space telescope", Proc. of SPIE, Space Telescopes and Instruments $V$ 3356, 1998.

2. D. R. Coulter, "Technology development for the next generation space telescope: an overview", Proc. of SPIE, Space Telescopes and Instruments $V$ 3356, 1998.

3. G. E. Mosier, M. Femiano, K. Ha, P. Y. Bely, R. Burg, D. C. Redding, A. Kissil, J. Rakoczy, L. Craig, "Integrated modeling environment for systems-level performance analysis of the next generation space telescope", Proc. of SPIE Space Telescopes and Instruments V 3356, 1998.

4. R. G. Lyon, J. E. Dorband, and J. M. Hollis, "Hubble Space Telescope Faint Object Camera calculated point-spread functions", Applied Optics 36, Number 8, pp. 1752-1765, March 10, 1997.

5. P. S. Davila, C. M. LeBoeuf, M. E. Wilson, E. W. Young, A. E. Lowman, D. C. Redding, "Optical design of the developmental cryogenic active telescope testbed", Proc. of SPIE, Space Telescopes and Instruments V 3356, 1998.

6. E. W. Young, M.E. Wilson, P. S. Davila, W. Eichhorn, C. M. LeBoeuf, D. C. Redding, A. E. Lowman, "Performance analysis of the developmental cryogenic active telescope testbed (DCATT)", Proc. of SPIE, Space Telescopes and Instruments $V$ 3356, 1998.

7. J. Hayes and S. Lange, "A heterodyne interferometer for testing laser diodes", Proc. Soc. Photo-Opt. Instrum. Eng. 429, pp. 22-26, 1983.

8. Y. Y. Cheng and J. C. Wyant, "Multiple-wavelength phase-shifting interferometry", Applied Optics 24, pp. 804-807, 1985.

9. L. K. Pacini, P. Geithner, D. N. Jacobson, D. C. Redding, "NEXUS: The next generation space telescope integrated flight systems demonstration", Proc. of SPIE, Space Telescopes and Instruments V 3356, 1998. 